\title{
Long-term transplant liver biliary access with a silastic ' $O$ ' tube
}

\author{
S Beningfield, MB ChB, FFRad (D) \\ Department of Radiology, University of Cape Town
}

Liver transplants are infrequently affected by serious biliary complications, including bile duct stenoses, biliary dilatation, intrahepatic stone formation and sepsis. ${ }^{1}$ In the face of immunosuppression, these problems can be especially problematic. Endoscopic or percutaneous routes are generally available, but if a hepaticojejunostomy has been performed, this precludes the endoscopic route. A method of permitting long-term access to the biliary tree without the need for drainage bags, catheter replacements, repuncture or surgery is described.

A middle-aged female patient underwent a liver transplant in 2000, but some months after the transplant developed clinical features of biliary complications. Imaging revealed bilateral duct dilation with intervening stenoses, and associated intrahepatic calculi (Fig. 1).

Initial standard percutaneous biliary access to the left and right bile ducts was obtained; firstly with internal-external drains, and later with internal stents. Multiple intraheptic calculi, focally dilated ducts and very fibrotic stenoses were encountered. The initial drainage catheters and stents repeatedly occluded and required regular replacement.

Consequently, it was decided to place a larger bore silastic tube across both left and right bile ducts, as a modified form of a $\mathrm{U}$ tube. ${ }^{2}$ Standard angiographic catheters and guidewires were introduced into left and right bile ducts; this allowed snaring of a guidewire from one side, permitting the through-and-through passage of the guidewire.

The long limb of a $12 \mathrm{~F}$ silastic T-tube was cut to approximately 40 $\mathrm{cm}$, and side-holes were added by cutting across the 'ears' created by kinking the tube at desired points. After dilatation, the $12 \mathrm{~F}$ silastic tubing was passed through the track over a coaxial catheter and guidewire, exiting on the opposite side (Fig. 2).

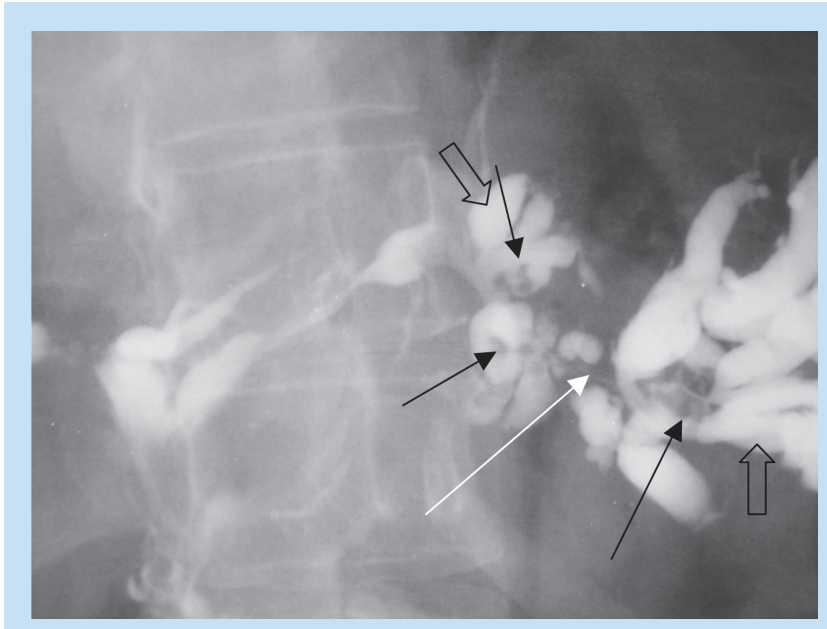

Fig. 1a. Close up of left-side cholangiogram showing abnormally dilated ducts (open arrows) with intervening short stenotic regions (white arrow), and intrahepatic stones (black arrows).

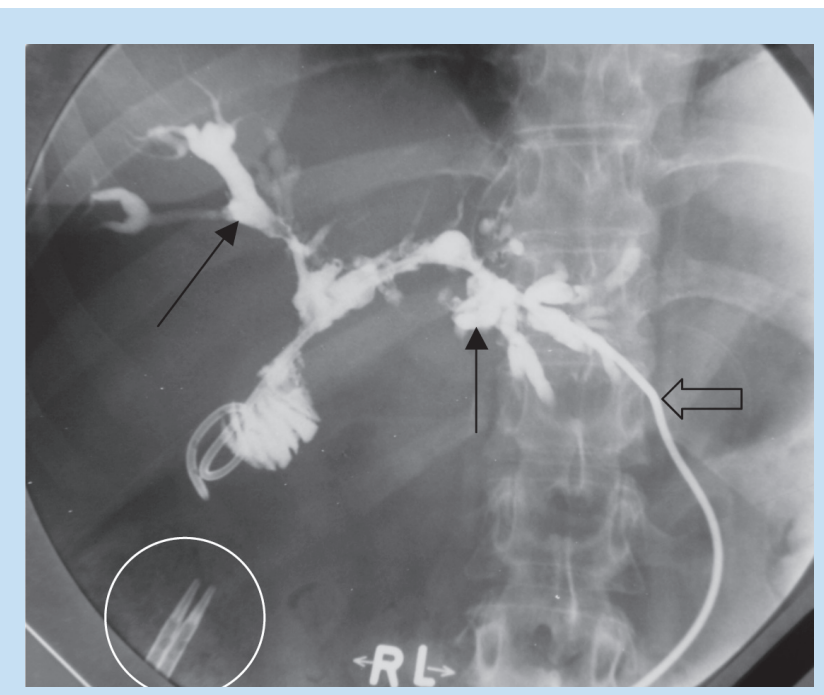

Fig. 1b. Cholangiogram showed abnormally diated ducts (arrows). Note left internal-external drain (open arrow) and displaced internal stents (white circle).

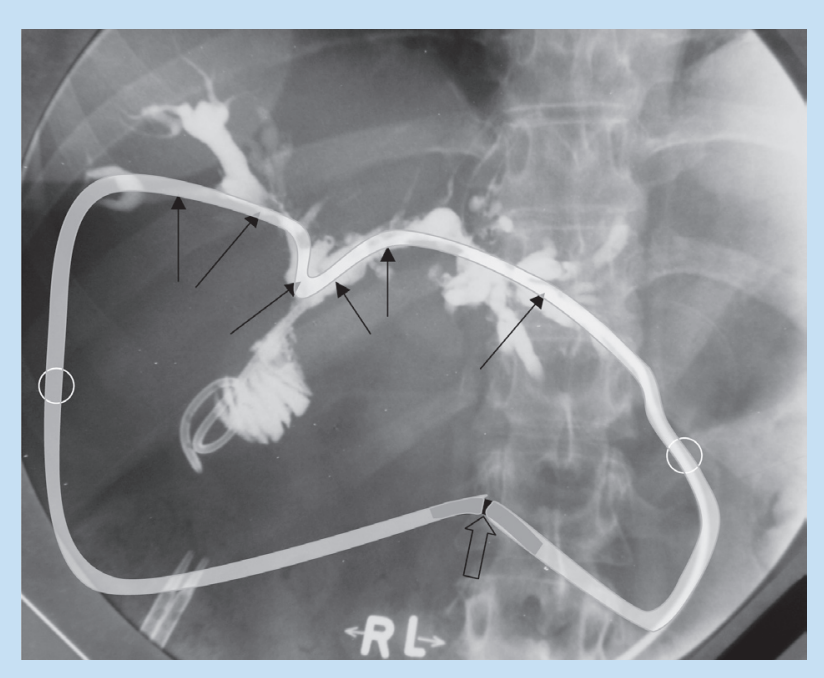

Fig. 2. Illustration of position of ' $O$ ' tube. Approximate side-hole positions (arrows); skin entry points (circles). Site of external join with short piece of catheter (open arrow).

The original tube has been in place for over 2 years, and has been cleaned and repositioned every three to six months. Tube cleaning is facilitated by passing a guidewire and catheter combination through the lumen, and rubbing the tube against the inner catheter/ guidewire. The system is also doused in povidone iodine, and washed thoroughly in saline thereafter. Biliary stone removal and stricture dilatation are undertaken while the silastic catheter is not in place.

The silastic catheter is then replaced over a guidewire, supplemented by a $5 \mathrm{~F}$ catheter placed internally to improve pushability. The two ends of the silastic catheter are joined to each other by pushing a $3 \mathrm{~cm} \mathrm{seg-}$ 


\section{SHORT REPORT}

ment of 9F Teflon transjugular catheter into either end.

The durability and lack of cracking, kinking or major encrustation of the silastic tube have been notable. The relatively large bore track has epithelialised well, and permits good access for stone removal and biliary dilatation techniques. The adjacent skin is healthy.

Although consideration has been given to removing the tube, the anxiety has been of recurrent stone reformation as a result of stagnation associated within the ductal ectasia and stenoses. Apart from access, the catheter also provides a stenting mechanism, limiting re-stenosis.

Silastic catheters have been used for long periods in the biliary tree for a number of years. ${ }^{3,4}$ Alternative forms of long-term biliary access include options such as internal-external drains and access loops. In cases where surgical creation of an access loop is not feasible, consideration can be given to use of a silastic tube system to allow ready biliary intervention on both left and right biliary trees. It should be noted that this system is suitable only where there is adequate internal drainage, either naturally or assisted by stents or other drains.

The benefits of the system described above include good patient tolerance, lack of a drainage bag and easy access to both sides of the biliary tree, without the need for repeat percutaneous punctures and catheter replacements.

Lake JR Long-term management of biliary tract complications. Liver Transpl Surg 1995; 1:45-54

Terblanche J, Louw JH. U tube drainage in the palliative therapy of carcinoma of the main hepatic duct junction. Surg Clin North Am 1973; 53:1245-1256.

Cameron JL, Gayler BW, Zuidema GD. The use of silastic transhepatic stents in benign and malignant biliary strictures. Ann Surg 1978; 188:552-561

4. Kolff J, Hoeltge G, Hermann RE. Silastic T tube splints for biliary repair. Am J Surg 1975; 129:236-240 $\underset{\substack{\text { EISEVIER } \\ \text { SAUNDERS }}}{\text { S }}$

KUMAR CLARK

CLINICAL MEDICINE SIXTH EDITION

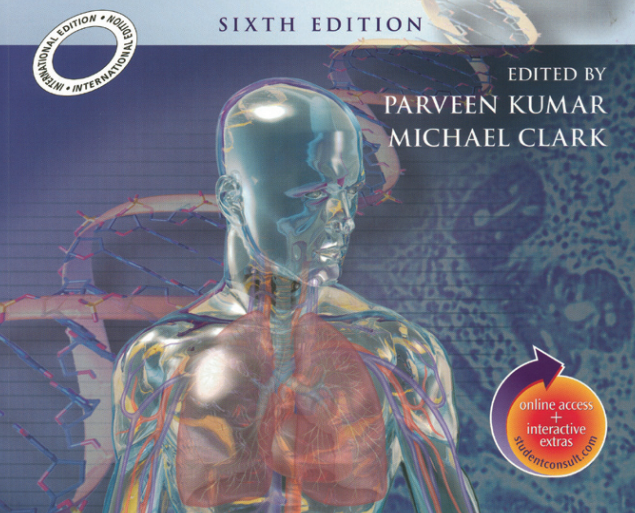

Health \& Medical Books

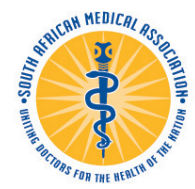

\section{SA Medical Association, Health \& Medical Publishing Group Private bag $X 1$, Pinelands, 7430 Tel: 021-6578200 - Fax: 021-6834509 e-mail: carmena@hmpg.co.za or brents@hmpg.co.za}

